\title{
Human studies with probiotics and prebiotics: clinical implications
}

\author{
J. M. Saavedra ${ }^{1,2 *}$ and A. Tschernia ${ }^{3}$ \\ ${ }^{1}$ Johns Hopkins University School of Medicine, 600 North Wolfe Street, Baltimore, MD 21287, USA \\ ${ }^{2}$ Nutrition Division, Nestlé USA, 800 North Brand Blvd., Suite 20, Glendale, CA 91203, USA \\ ${ }^{3}$ Division of Pediatric GI, Nutrition and Liver Diseases, Mount Sinai School of Medicine, New York, NY, USA
}

\begin{abstract}
Probiotic agents have been shown to have significant clinical beneficial effects in the prevention and management of gastrointestinal and non-gastrointestinal conditions. These observations have led to work demonstrating that an important mechanism of these agents is their close interaction with the gut associated lymphoid tissue (GALT) and suggested immunomodulatory effects on systemic immune response. Studies on the possibility that prebiotic agents might directly or indirectly induce similar immunomodulation have only recently begun. The preliminary findings of several recent human clinical trials reviewed in this article indicate that prebiotics may indeed prove to be a clinically beneficial dietary supplement, in the context of novel nutritional strategies for the management of gastrointestinal and systemic conditions.
\end{abstract}

Prebiotics: Immunology: Fructo-oligosaccharides: Oligofructose: Probiotics

\section{Introduction}

The intestinal milieu constitutes the setting for close interaction between the individual and its surrounding environment, with a constant exchange of energy, water, nutrients and electrolytes. The mucosal surface of the gut serves as an effective barrier to prevent entry of a wide spectrum of pathogens, toxins and allergens; however in clear contrast with other mucosal organs, the gut must allow for the passage of nutrients. A complex ecosystem, the intestinal flora actively interrelates with this mucosal surface. The largest lymphoid organ in the body, the gut associated lymphoid tissue (GALT) evolves and responds dynamically to the presence of and changes in the intestinal flora (Drasar \& Roberts, 2001). Lack of this 'microbial experience' leads to significant pathophysiological consequences. For example, an underdeveloped GALT in animals devoid of an intestinal flora results in persistent enteritis, severe infections and poor survival. In addition, a metabolically active intestinal flora is critical for the maintenance of a healthy gut epithelium, vitamin production, bile acid metabolism and enterohepatic circulation to name a few. Additionally changes in the human microflora have been correlated with modulated local and systemic immune responses of the GALT (De Simone et al. 1992; LinkAmster et al. 1994).

\section{Probiotics and immunological effects}

The concept of probiotics has advanced rapidly over the last several years. The body of circumstantial evidence suggesting immunomodulatory properties in particular is today vast and varied: the purposeful modification of the intestinal microbiota with probiotics leads to select clinical beneficial effects (Fuller, 1991; Hanson \& Yolken, 1999; Naidu et al. 1999; Elmer et al. 1996). Such specific effects with ingestion of particular strains have been demonstrated; for example the ingestion of lactase producing lactobacillus decreased symptoms of lactose maldigestion (Shermak et al. 1995). The use of probiotics has been shown to have a therapeutic as well as prophylactic effect on diarrhoeal illnesses of multiple aetiologies such as Clostridium difficile induced enteritis (Biller et al. 1995; Gorbach et al. 1987), traveller's diarrhoea (Hilton et al. 1997) as well as antibiotic associated diarrhoea (Vanderhoof et al. 1999). Several well-controlled studies have demonstrated that dietary supplementation with either bifidobacteria or lactobacilli will decrease the duration and/or severity of acute diarrhoeal disease in infants and children (Guarino et al. 1997; Majamaa et al. 1995; Oberhelman et al. 1999). Prospective studies have also shown that the incidence of acute diarrhoea can be decreased (Saavedra et al. 1994; 1999a,b). Interestingly however, the best

\footnotetext{
Abbreviations: GALT, gut associated lymphoid tissue.

Note: For the definition of the terms inulin and oligofructose please refer to the introductory paper (p. S139) and its footnote.

*Corresponding author: Professor J. M. Saavedra, fax +1 818549 5704, email jose.saavedra@us.nestle.com
} 
documented effects in diarrhoeal disease both in animals and humans have been observed in cases of viral gastroenteritis (Duffy et al. 1994; Saavedra et al. 1999b). The supplementation with probiotics in infants with atopic dermatitis and cow's mild protein allergy has also shown positive outcomes (Pessi et al. 2000; Majamaa \& Isolauri, 1997). The probiotic effect is unlikely to be due a simple overwhelming of indigenous flora by the ingested nonpathogenic organism. Numerous studies now suggest direct probiotic immunomodulation of the GALT, its humoral as well as its cellular immune component (Isolauri et al. 1995; Kaila et al. 1992; Marteau \& Cellier, 1999; Perdigon et al. 1994). The mechanisms for this immunomodulation are still unknown as of this writing; probiotic agents can induce cytokine production in varying degrees, including intraleukin 12 , tumor necrosis factor alpha and interferon gamma (IL-12, TNF-K, and IFN-3) (Solis \& Lemonnier, 1991) increased production of IFN alpha in macrophages, mediated by messenger RNA encoding IFN-K. (Kitazawwa et al. 1994), Peyer patch proliferation of B-cells, with enhanced antibody production (Yasui \& Ohwaki, 1991; Yasui et al. 1994), and optimization of cellular immune responses (Chiang et al. 2000).

Animal studies with Bifidobacterium bifidum have shown that several probiotics may have a beneficial effect against rotaviral diarrhoea (Duffy et al. 1993; 1994). This is a systemic response (Yasui et al. 1995), as demonstrated by the passive protection against rotavirus observed in mouse pups born to and nursed by dams fed bifidobacteria. This protection was mediated through an increase in the amount of specific anti rotavirus IgA in the milk of dams fed probiotics. In mammals, including humans, the entero-mammary pathway for secretory $\operatorname{Ig} \mathrm{A}$ production is thought to be an important protective mechanism against infections in breast-fed children. Finally, recent studies show that additional mechanisms induced by probiotic ingestion may play a role in protection from pathogens, such as decreasing adherence by inducing the secretion of specific mucins through induction of MUC genes in the gut (Mack et al. 1999), thus modulating the barrier effect of the gut.

\section{Prebiotics and immunological effects}

Of particular interest has been the fact that breast-fed infants carry bifidobacteria as their predominant intestinal flora, with wide variations amongst different populations (Balmer \& Wharton, 1989; Yoshioka et al. 1983). This native probiotic infant flora is maintained in the breast-fed infant in part via bifidogenic substrates, galacto-oligosaccharides (McVeagh \& Miller, 1997), contained in human breast milk and classically termed 'bifido factor'. This interplay between flora and breastmilk has drawn attention to the bifidogenic effect of nutritional supplements and bifidogenicity has become a hallmark of the prebiotic concept.

Oligofructose and other oligosaccharides have a significant effect on the population of luminal flora, in particular, stimulating bifidobacterial populations (Bouhnik et al. 1997; Gibson et al. 1995; 1999; Rao, 1999; Roberfroid, 1996). Bifidobacteria are present in high counts early in the infant faecal flora, but the numbers of these native organisms decreases significantly thereafter. Oligofructose as a prebiotic has shown specific bifidogenicity. Thus, it is likely that the immunological effects observed in probiotic studies could be demonstrated via the use of prebiotics, and that these agents may ultimately demonstrate similar clinical benefits as have been reported with probiotic bacteria. Only recently have these efforts begun, but the initial observations appear very promising.

\section{Human prebiotic clinical trials}

Human studies have only recently begun. Although most of the information discussed here has been only recently presented or is preliminary, the data suggest clinically significant effects that warrant further study and explanation.

We recently presented the results of a double masked, randomized controlled study to assess several clinical parameters related to common acute paediatric illnesses in infants and toddlers, supplemented with oligofructose. The gastro-intestinal tolerance to this oligofructose, a non-absorbable non-digested carbohydrate was also assessed (Saavedra et al. 1999; Tschernia et al. 1999).

One hundred and twenty-three out of 140 subjects recruited completed the study. These healthy children, between the ages of 4 and 24 months were already consuming cereal prior to enrolment in the study and were all attending daycare in a large metropolitan area. The children were randomized to receive a commercially available infant cereal alone or the same cereal supplemented with oligofructose at a concentration of $0.55 \mathrm{~g}$ per $15 \mathrm{~g}$ of dry cereal.

The cereal was offered ad libitum, with an encouraged minimum goal intake of $15 \mathrm{~g}$ serving of dry cereal per day.

Total consumption was calculated using data obtained both at home and at the daycare. Once enrolled, children remained on the study as long as they continued to consume cereal. Data was obtained through a weekly daycare visit and a weekly phone call with a parent or guardian, using a standardized questionnaire and included: height and weight, cereal consumption, bowel movement frequency and consistency, GI symptomatology (regurgitation, flatulence, apparent discomfort) and diaper rash. When the caretaker perceived 'diarrhoea', further information was queried pertaining to stool frequency, consistency and duration of perceived illness as well as associated symptoms fever, vomiting and pain. Parents were also queried for the presence of cold symptoms, with particular attention to the presence of fever and use of antibiotics.

Both groups showed similar cereal consumption (average $3.32 \mathrm{~g} / \mathrm{kg}$ per day supplemented, $3.45 \mathrm{~g} / \mathrm{kg}$ per day control). During the entire study period, the supplemented group consumed a calculated daily average of $1.1 \mathrm{~g}$ oligofructose/day; all subjects exhibited normal growth during the study, and the cereal was well tolerated in both groups.

No significant differences were observed when comparing the occurrence of perceived flatulence, stool frequency and consistency. A significantly lower frequency of reported emesis, regurgitation (spitting up), and perceived discomfort with bowel movements was noted in the supplemented group. The supplemented group reported a fre- 
quency of constipation of almost $40 \%$ less than the unsupplemented group, although this did not reach statistical significance (Fig. 1).

The overall incidence of diarrhoea by parental report was not significantly different between the two groups. This remained the case when a more stringent, albeit arbitrary, definition of diarrhoea was applied (daily loose or watery stool frequency greater than two and lasting for at least $48 \mathrm{~h}$ ). Using this definition, the supplemented and control groups had 2.5 and 2.08 episodes per subject year (not significant). There were no statistical differences in stool consistency during these events and the average duration of a diarrhoeal episode was similar (2.97 days in supplemented group v. 3.37 days in the control groups, no significance). In this healthy population, diarrhoeal illness was mild in terms of purge, and the impact on hydration status was minimal. No subject was hospitalized for diarrhoeal disease during the entire study period.

However, highly significant differences were noted when comparing the groups for the occurrence of concurrent fever as an indicator of severity during diarrhoeal episodes (15.7\% supplemented v. $40.4 \%$ control, $P<0.05)$. A similar and highly significant decrease in medical attention seeking during such an event was also detected in the oligofructose-supplemented group (16.13\% v. control $24.3 \%$, $P<0.05)$. A greater daycare absenteeism rate during reported diarrhoea was observed in the control group (supplemented 0.31 days per subject-week of diarrhea, control 0.79 days per subject-week of diarrhoea), Fig. 2. A significant difference in the occurrence of fever with 'cold symptoms' between the two groups was also noted (supplemented $5.76 v$. control 9.05 episodes per subject year, $P<0 \cdot 05)$. Additionally, the control subjects reported a significantly higher use of antibiotics during respiratory illness (8.33 v. $12 \cdot 03$ episodes per subject year, $P<0.05)$.
This first study on the clinical benefits of oligofructose supplementation clearly demonstrates an interesting impact on the occurrence of febrile illness, either associated with diarrhea or upper respiratory illness; both are usually of viral etiology in the pediatric population. The question of potential immunomodulatory activity through this novel nutritional prebiotic strategy is raised.

A recent study by Firmansyah et al. (2001) seems to add further weight to this observation. The authors studied the effect of a cereal weaning food, supplemented with oligofructose on the immunological response to measles vaccine. In this double masked, placebo controlled trial, fifty healthy infants aged 7-9 months of age were randomized to receive a standard cereal or one supplemented with a mixture of oligofructose and inulin, added at a concentration of $1 \mathrm{~g}$ per $25 \mathrm{~g}$ of dry weight cereal.

The subjects received one to two servings of the cereals daily for 10 weeks. After 4 weeks of participation, the children were immunized using a standard live attenuated measles vaccination. Data collected included weight and height at baseline, at week 4 and again at week 10. Antibody levels (anti measles $\operatorname{IgG}$ and $\operatorname{IgM}$ ) were measured by ELISA method at baseline and at week 10. Interview at weeks 4, 6 and 10 of participation were conducted and data collected included cereal intake, stool frequency and consistency, flatulence and vomiting. Statistical analysis did not reveal any differences between the groups at baseline, and both cereals were well tolerated. Importantly there was no difference on growth parameters between groups during the entire study. Mean intake of cereal was $5 \mathrm{~g}$ per $\mathrm{kg}$ body weight per day, with no difference between the two groups. The supplemented group received $0.2 \mathrm{~g}$ prebiotic per $\mathrm{kg}$ body weight per day.

However a significant difference was noted when comparing anti measles IgG: initial levels were low and similar

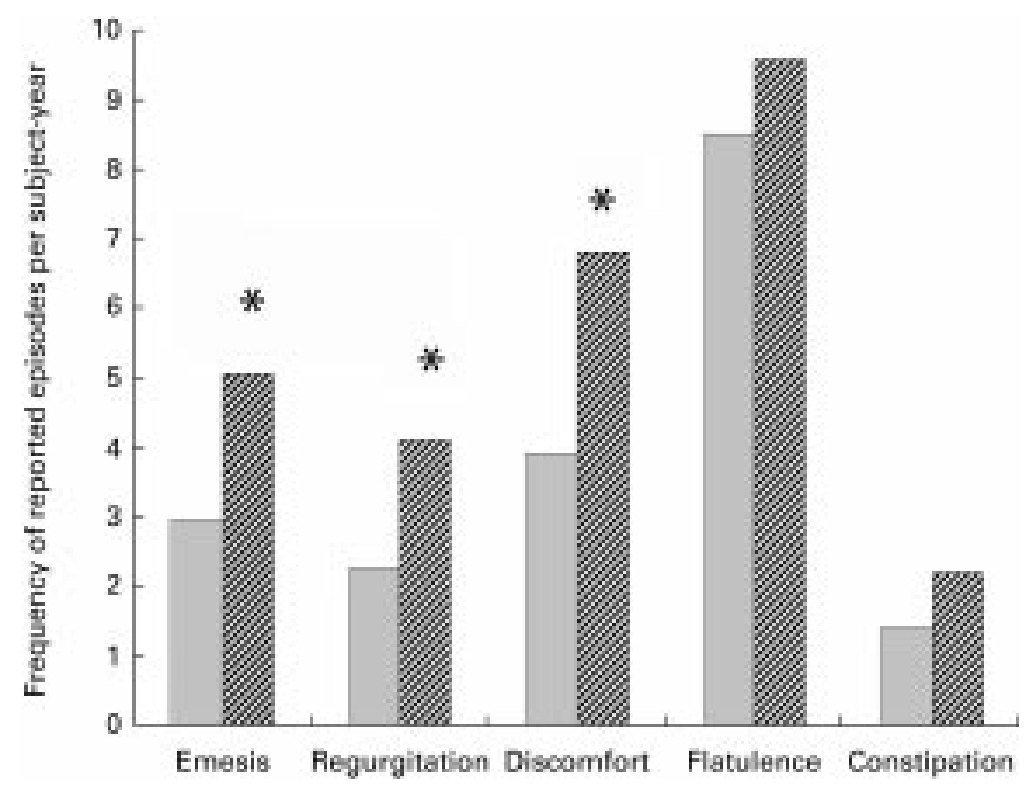

Fig. 1. Reported frequency of gastrointestinal related symptoms over the study period. $\quad=$ group supplemented with oligofructose. $=$ control group. ${ }^{\star} P<0.05$. Adapted from Saavedra et al. (1999) and Tschernia et al. (1999). 


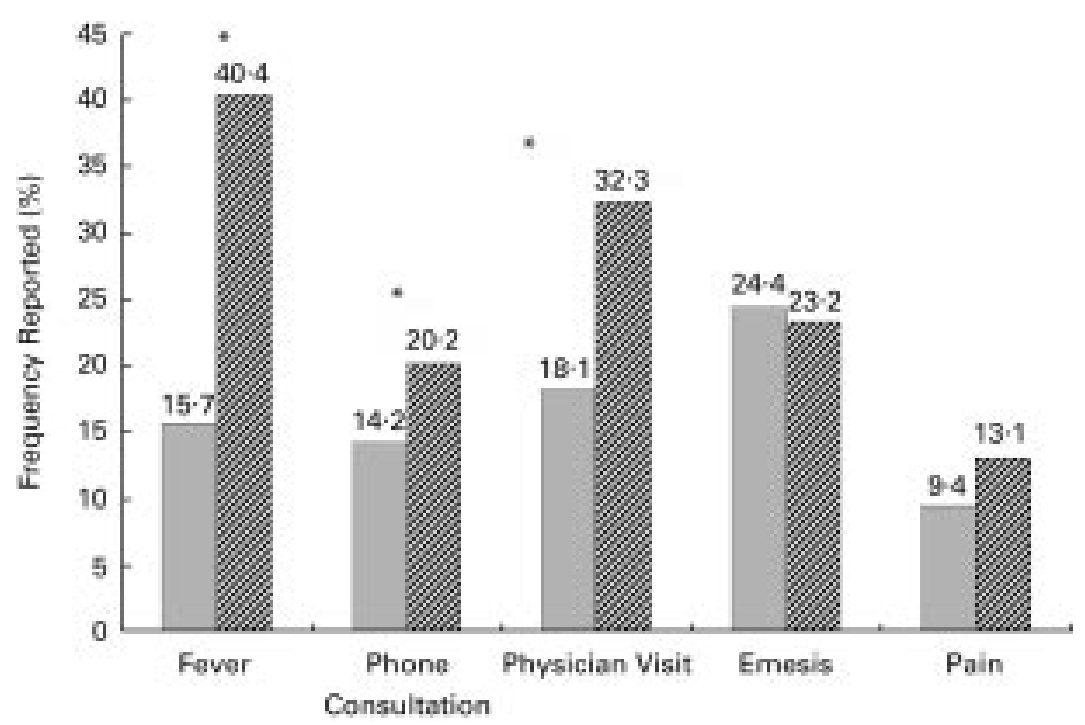

Fig. 2. Reported frequency (\% of times) specific signs, symptoms or events were associated with diarrhoea. = group supplemented with oligofructose. = control group. ${ }^{*} P<0.05$. Adapted from Saavedra et al. (1999) and Tschernia et al. (1999).

between groups at baseline, but a significant difference post-immunization was clearly detected above baseline (supplemented group: 6.6-fold increase $v$. control: 4.2fold increase, $P<0.03$ ). Anti measles IgM levels showed no inter- or intragroup significant differences throughout the study. The positivity rates for children with adequate IgG antibody response were $96 \%$ and $88 \%$ in the supplemented and control group, respectively $(P<0 \cdot 01)$. Mild reactions in the period post vaccination were observed more frequently in the supplemented group. Although this study does not provide any further insight into the mechanism of the observed clinical benefit, a targeted nutritional strategy using prebiotics during childhood immunization certainly deserves further attention and investigation.

Finally, two other recent studies using a synbiotic, the concurrent administration of a probiotic and a prebiotic have shown significant effects. In a double masked trial (Ahmad et al. 2000), fifty-eight healthy children with acute gastroenteritis were randomized to receive a low lactose formula with or without a combination of Lactobacillus rhamnosus and oligofructose. The duration of diarrhoea was significantly shortened in the supplemented group (1.63 v. 2.45 days).

The second study, conducted by Fisberg et al. (2000) was a multicenter, double masked controlled trial: 626 children, aged between 1 and 6 years with mild to moderate malnutrition were enrolled to receive a nutritional supplement with or without a synbiotic preparation (containing Lactobacillus acidophilus, Bifidobacterium infantis and oligofructose). Children were evaluated by a monthly visit, for 4 months, and data recorded included supplement intake, height, weight, and stool pattern incidence and duration of illness, and episodes requiring antibiotics. The number of 'sick days' for subjects aged 3-5 years was significantly lower in the group receiving the synbiotic supplement. Overall the total number of 'sick days' decreased in both groups over the study time, as the nutritional status of these children improved.

Although the latter two studies used the combination of a prebiotic and a probiotic, they suggest that even in high risk, ill pediatric populations oligofructose is well tolerated, and may be partially responsible for the clinical beneficial effects noticed.

Further studies will be necessary to corroborate these preliminary findings. As we have demonstrated, infants and young children tolerate oligofructose, up to $0.8 \mathrm{~g} / \mathrm{kg}$ body weight per day, in our own experience. In none of these controlled trials were there any significant differences in the potential signs of gastrointestinal intolerance such as flatulence, abdominal discomfort, or increased purge as noted in adult studies typically with doses above $15 \mathrm{~g} / \mathrm{d}$. Of note, most of these pediatric studies utilized semi-solid foods (cereal) as a vehicle for administration; which may be a major factor in allowing for the delivery of higher doses without adverse symptoms.

Thus far, the pediatric studies available using exclusively a prebiotic preparation have been carried out in healthy populations. These children typically will have fewer disruptions in the intestinal flora, and a potential better bifidogenic response than in children in developing countries, with high morbidity or high nutritional risk. In this latter population, a synbiotic may prove more efficacious than either agent alone.

The mechanisms of action certainly need to be adequately defined, but it is becoming apparent that the modifications of intestinal flora by the ingestion of probiotics, prebiotics or synbiotics can interact with the immunological component of the intestine, and yield not only gastrointestinal protective effects, but given the nature of the immune response of the GALT, may yield systemic effects, that in turn may have significance in other mucosal 
surfaces, such as the skin and respiratory tract, thereby providing a broader systemic benefit. Much has been learned, but much has yet to be learned.

\section{References}

Ahmad A, Widjala L, Firmansyah A, Gliwitzki M \& Suhardjo H (2000) Effect of a combined probiotic, prebiotic and micronutrient supplementation in reducing duration of acute infantile diarrhoea. Journal of Paediatric Gastroenterology and Nutrition 31, A984.

Balmer SE \& Wharton BA (1989) Diet and faecal flora in the newborn: breast milk and infant formula. Archives of Diseases in Childhood 64, 1672-1677.

Biller JA, Katz AJ, Flores AF, Buie TM \& Gorbach SL (1995) Treatment of recurrent Clostridium difficile colitis with Lactobacillus GG. Journal of Paediatric Gastroenterology and Nutrition 21, 224-226.

Bouhnik Y, Flourie B, D’Agay-Abensour L, Pochart P, Gramet G, Durand M \& Rambaud JC (1997) Administration of transgalacto-oligosaccharides increases fecal bifidobacteria and modifies colonic fermentation metabolism in healthy humans. Journal of Nutrition 127, 444-448.

Chiang BL, Sheih YH, Wang LH, Liao CK \& Gill HS (2000) Enhancing immunity by dietary consumption of a probiotic lactic acid bacterium (Bifidobacterium lactis HN019): optimization and definition of cellular immune responses. European Journal of Clinical Nutrition 54, 849-855.

De Simone C, Ciardi A, Grassi A, Lambert GS, Tzantzoglou S, Trinchieri V, Moretti S \& Jirillo E (1992) Effect of Bifidobacterium bifidum and Lactobacillus acidophilus on gut mucosa and peripheral blood B lymphocytes. Immunopharmacology Immunotoxicology 14, 331-340.

Drasar B \& Roberts A (2001) Control of the large bowel microflora. In Human Microbial Ecology, pp. 87-111 [M Hill and P Marsh, editors]. Boca Raton, FL: CRC Press.

Duffy LC, Zielezny MA, Riepenhoff-Talty M, Dryja D, SayahtaheriAltaie S, Griffiths E, Ruffin D, Barrett H, Rossman J \& Ogra PL (1994) Effectiveness of Bifidobacterium bifidum in mediating the clinical course of murine rotavirus diarrhea. Pediatric Research 35, 690-695.

Duffy LC, Zielezny MA, Riepenhoff-Talty M, Dryja D, SayahtaheriAltaie S, Griffiths E, Ruffin D, Barrett H, Rossman J \& Ogra PL (1993) Effectiveness of Bifidobacterium bifidum in experimentally induced MRV infection: dietary implications in formulas for newborns. Endocrine Regulation 27, 223-229.

Elmer GW, Surawicz CM \& McFarland LV (1996) Biotherapeutic agents. A neglected modality for the treatment and prevention of selected intestinal and vaginal infections. Journal of American Medical Association 275, 870-876.

Firmansyah A, Pramita G, Carrie Fassler A, Haschke F \& LinkAmster H (2001) Improved humoral immune response to measles vaccine in infants receiving infant cereal with fructooligosaccharides. Journal of Paediatric Gastroenterology and Nutrition 31, A521.

Fisberg M, Maulen I, Vasquez E, Garcia J, Comer G \& Alarcon P (2000) Effect of oral supplementation with and without synbiotics on catch-up growth in preschool children. Journal of Paediatric Gastroenterology and Nutrition 31, A987.

Fuller R (1991) Probiotics in human medicine. Gut 32, 439-442. Gibson GR (1999) Dietary modulation of the human gut microflora using the prebiotics oligofructose and inulin. Journal of Nutrition 129, suppl, 1438S-1441S.

Gibson GR, Beatty ER, Wang X \& Cummings JH (1995) Selective stimulation of bifidobacteria in the human colon by oligofructose and inulin. Gastroenterology 108, 975-982.
Gorbach SL, Chang TW \& Goldin B (1987) Successful treatment of relapsing Clostridium difficile colitis with Lactobacillus $G G$. Lancet 2, 8574, 1519.

Guarino A, Canani RB, Spagnuolo MI, Albano F \& Di Benedetto L (1997) Oral bacterial therapy reduces the duration of symptoms and of viral excretion in children with mild diarrhea. Journal of Paediatric Gastroenterology and Nutrition 25, $516-519$.

Hanson L \& Yolken R (1999) Probiotics, other nutritional factors and intestinal microflora. Philadelphia: Lippincott-Raven Publishers.

Hilton E, Kolakowski P, Singer C \& Smith M (1997) Efficacy of Lactobacillus $G G$ as a diarrheal preventive in travelers. Journal of Travel Medicine 4, 41-43.

Isolauri E, Joensuu J, Suomalainen H, Luomala M \& Vesikari T (1995) Improved immunogenicity of oral D $\times$ RRV reassortant rotavirus vaccine by Lactobacillus casei GG. Vaccine 13, $310-312$.

Kaila M, Isolauri E, Soppi E, Virtanen E, Laine S \& Arvilommi H (1992) Enhancement of the circulating antibody secreting cell response in human diarrhea by a human Lactobacillus strain. Pediatric Research 32, 141-144.

Kitazawa H, Tomioka Y, Matsumura K, Aso H, Mizugaki M, Itoh T \& Yamaguchi T (1994) Expression of mRNA encoding IFN alpha in macrophages stimulated with Lactobacillus gasseri. FEMS Microbiology Letters 120, 315-321.

Link-Amster H, Rochat F, Saudan KY, Mignot O \& Aeschlimann JM (1994) Modulation of a specific humoral immune response and changes in intestinal flora mediated through fermented milk intake. FEMS Immunology \& Medical Microbiology 10, 55-63.

Mack DR, Michail S, Wei S, McDougall L \& Hollingsworth MA (1999) Probiotics inhibit enteropathogenic E. coli adherence in vitro by inducing intestinal mucin gene expression. American Journal of Physiology 276, G941-G950.

Majamaa H \& Isolauri E (1997) Probiotics: a novel approach in the management of food allergy. Journal of Allergy and Clinical Immunology 99, 179-185.

Majamaa H, Isolauri E, Saxelin M \& Vesikari T (1995) Lactic acid bacteria in the treatment of acute rotavirus gastroenteritis. Journal of Paediatric Gastroenterology and Nutrition 20, 333-338.

Marteau P \& Cellier C (1999) Immunological effects of biotherapeutic agents. In Biotherapeutic Agents and Infectious Diseases, pp. 121-144 [GW Elmer, LV McFarland and CM Surawicz, editors]. Totowa, NJ: Humana Press.

McVeagh P \& Miller JB (1997) Human milk oligosaccharides: only the breast. Journal of Paediatrics and Child Health 33, 281-286.

Naidu A, Bidlack W \& Clemens R (1999) Probiotic spectra of lactic acid bacteria. Critical Reviews in Food Science and Nutrition 39, 13-126.

Oberhelman RA, Gilman RH, Sheen P, Taylor DN, Black RE, Cabrera L, Lescano AG, Meza R \& Madico G (1999) A placebo-controlled trial of Lactobacillus $G G$ to prevent diarrhea in undernourished Peruvian children. Journal of Paediatrics 134, 15-20.

Perdigon G, Rachid M, De Budeguer MV \& Valdez JC (1994) Effect of yogurt feeding on the small and large intestine associated lymphoid cells in mice. Journal of Dairy Research 61, $553-562$.

Pessi T, Sutas Y, Hurme M \& Isolauri E (2000) Interleukin-10 generation in atopic children following oral Lactobacillus rhamnosus GG. Clinical and Experimental Allergy 30, 1804-1808.

Rao AV (1999) Dose-response effects of inulin and oligofructose on intestinal bifidogenesis effects. Journal of Nutrition 129, suppl, 1442S-1445S. 
Roberfroid MB (1996) Functional effects of food components and the gastrointestinal system: chicory fructo-oligosaccharides. Nutrition Reviews 54, S38-S42.

Saavedra JM \& Abi-Hanna A (1999a) Clinical studies of probiotic agents. In Probiotics, Other Nutritional Factors and Intestinal Microflora, pp. 271-286 [L Hanson and RH Yolken, editors]. Philadelphia: Lippincott-Raven.

Saavedra JM, Bauman NA, Oung I, Perman JA \& Yolken RH (1994) Feeding of Bifidobacterium bifidum and Streptococcus thermophilus to infants in hospital for prevention of diarrhoea and shedding of rotavirus. Lancet 344, 8929, 1046-1049.

Saavedra JM, Tschernia A, Moore N, Abi-Hanna A, Coletta F, Emenhiser C \& Yolken R (1999b) Gastrointestinal function in infants consuming a weaning food supplemented with oligofructose a prebiotic. Journal of Paediatric Gastroenterology and Nutrition 29, A95.

Shermak MA, Saavedra JM, Jackson TL, Huang SS, Bayless TM \& Perman JA (1995) Effect of yogurt on symptoms and kinetics of hydrogen production in lactose-malabsorbing children. American Journal of Clinical Nutrition 62, 1003-1006.

Solis PB \& Lemonnier D (1991) Induction of $2^{\prime}-5^{\prime}$ A synthetase activity and interferon in humans by bacteria used in dairy products. European Cytokine Network 2, 137-140.
Tschernia A, Moore N, Abi-Hanna A, Yolken RH, Coletta F, Emenhiser C \& Saavedra JM (1999) Effects of long-term consumption of a weaning food supplemented with oligofructose, a prebiotic, on general infant health status. Journal of Paediatric Gastroenterology and Nutrition 29, A58.

Vanderhoof JA, Whitney DB, Antonson DL, Hanner TL, Lupo JV \& Young RJ (1999) Lactobacillus $G G$ in the prevention of antibiotic-associated diarrhea in children. Journal of Paediatrics 135, 564-568.

Yasui H, Kiyoshima J \& Ushijima H (1995) Passive protection against rotavirus-induced diarrhea of mouse pups born to and nursed by dams fed Bifidobacterium breve YIT4064. Journal of Infectious Disease 172, 403-409.

Yasui H, Nagaoka N \& Hayakawa K (1994) Augmentation of anti-influenza virus hemagglutinin antibody production by Peyer's patch cells with Bifidobacterium breve YIT4064. Clinical Diagnosis Laboratory Immunology 1, 244-246.

Yasui H \& Ohwaki M (1991) Enhancement of immune response in Peyer's patch cells cultured with Bifidobacterium breve. Journal of Dairy Science 74, 1187-1195.

Yoshioka H, Iseki K \& Fujita K (1983) Development and differences of intestinal flora in the neonatal period in breast-fed and bottle-fed infants. Pediatrics 72, 317-321. 